\title{
ADVANCEMENT OF VEHICLE OCCUPANT RESTRAINT SYSTEM DESIGN BY INTEGRATION OF ARTIFICIAL INTELLIGENCE TECHNOLOGIES
}

\author{
HIROSUKE HORII \\ Kokushikan University, Japan
}

\begin{abstract}
In order to improve the design method of vehicle occupant restraint systems, it is necessary to reduce the computational load of simulations, to improve the global search capability, and to examine and integrate analytical methods to understand the complex interaction between design variables and objective functions. Therefore, in this study, we integrated the following three artificial intelligence technologies and applied them to the design of a vehicle occupant restraint system: (1) construction of a highly accurate approximate model by machine learning, (2) improvement of global search capability by evolutionary multi-objective optimization and (3) visualization and knowledge acquisition of multidimensional information using multivariate analysis methods. First, we obtained the minimum number of actual calculation samples using a crash analysis model with the design of experiments, and then used these samples to construct a highly accurate approximate model using machine learning. Next, we used the approximate model to perform a global search in the design space by evolutionary multi-objective optimization to obtain a Pareto solution set that takes into account the trade-off relationship between the objective functions. Finally, multivariate analysis using cluster analysis and self-organizing maps was performed on the Pareto solution set. As a result, a fast global search was realized by substituting the evaluation calculation of evolutionary multi-objective optimization with a highly accurate approximate model. The Pareto solution set obtained therein was then partitioned into clusters by cluster analysis, and the partitioned clusters were analysed by self-organizing maps, which provided perceptual information on the factors governing the trade-offs between the objective functions and the interactions between the design variables, and were useful for design engineers' insights.
\end{abstract}

Keywords: cluster analysis, evolutionary computation, machine learning, multi-objective optimization, self-organizing maps, vehicle occupant restraint system.

\section{INTRODUCTION}

In the design of vehicle occupant restraint systems, it is necessary to consider the complex interaction of many design variables such as airbag and seatbelt operation control, interior equipment design such as seats, occupant's body size and posture, and crash conditions. In addition, there is a trade-off between improving the safety of each part of the human body, such as the head and chest, and it can be regarded as a multi-objective optimization problem in a global search space.

On the other hand, due to the high computational cost of virtual prototyping by computer simulations as well as the cost of actual prototyping, local search around the initial design or with narrowed design variables is generally used nowadays. In addition, with the development of research on optimization methods and the expansion of their application to realworld problems, it is considered insufficient to simply search for and present the optimal solution, and the importance of using the results obtained in the computational process to extract design information and visually and mathematically understand the interaction relationship between inputs and outputs has been proposed.

From the above point of view, Multi-objective Design Exploration [1], which structures and visualizes the knowledge about the design space based on evolutionary computation, Computer Aided Principle (CAP) [2], which uses design of experiments and cluster analysis 
to understand design information, and Innovization [3], which extracts design information from the results obtained in the computational process after multi-objective optimization, have been proposed so far.

In order to improve the design method of vehicle occupant restraint systems, we considered that it is necessary to reduce the computational load of simulation and to improve the global search capability, and to investigate the analysis method to understand the complex interaction between design variables and objective functions, and to integrate them.

Based on the above ideas, this paper reports the results of integrating the following three artificial intelligence technologies and applying them to the design of a vehicle occupant restraint system.

(1) Construction of a highly accurate approximate model by machine learning;

(2) Improvement of global search capability by evolutionary multi-objective optimization;

(3) Visualization and knowledge acquisition of multidimensional information using multivariate analysis methods.

\section{CONSTRUCTION OF CRASH ANALYSIS MODEL}

In order to evaluate the performance of vehicle occupant restraint systems, an analytical model was constructed assuming a full-wrap frontal crash test as shown in Fig. 1. This analytical model was employed as a basic model for the safety evaluation at an automotive company. In the actual development of the system, physical crash tests were carried out on the vehicles in question to calibrate their analytical model's specific physical characteristics. The structure of the developed frontal crash analysis model and the evaluation index of occupant safety performance are described below.

\subsection{Structure of a frontal crash analysis model}

In the full-wrap crash test, the impact on the head, chest and lower limbs of the crash test dummy is measured to evaluate its safety performance. The analytical model consists mainly of a multi-body model of the crash test dummy, the equipment around the occupant such as the seat, steering wheel and knee bolster, and the occupant restraint system of the airbag and seatbelt. Six variables, variables that control the operation of the airbag and seatbelt and variables that control the rigidity of the knee bolster are used as design variables. The acceleration waveform of a full-wrap frontal crash test is given to the analytical model to simulate the occupant behaviour for $0.12 \mathrm{~s}$ after the crash.

The design variables are shown in Table 1 . In the airbag, the inflator is triggered $A B \_T T F$ seconds after crash detection to deploy the airbag, and the deployment speed is controlled by the mass flow coefficient $A B \_M F R$. The efficiency of gas release from the airbag after deployment is controlled by the vent hole diameter setting factor $A B_{-} V H F$. In the seat belt, the pretensioner mechanism is activated after $S B \_T T F$ seconds of collision crash detection, and the load limiter is set to $S B \_L L(\mathrm{~N})$. The stiffness coefficient of the knee bolster is defined as $K B \_S F$.

\subsection{Evaluation index of occupant safety performance}

The head injury criterion (HIC), the chest resultant acceleration (ChestG), and the femoral compressive load are calculated based on the Japanese safety standards. In the following design, the $H I C$ and the Chest $G$, which have a large impact on occupant safety, are used as 


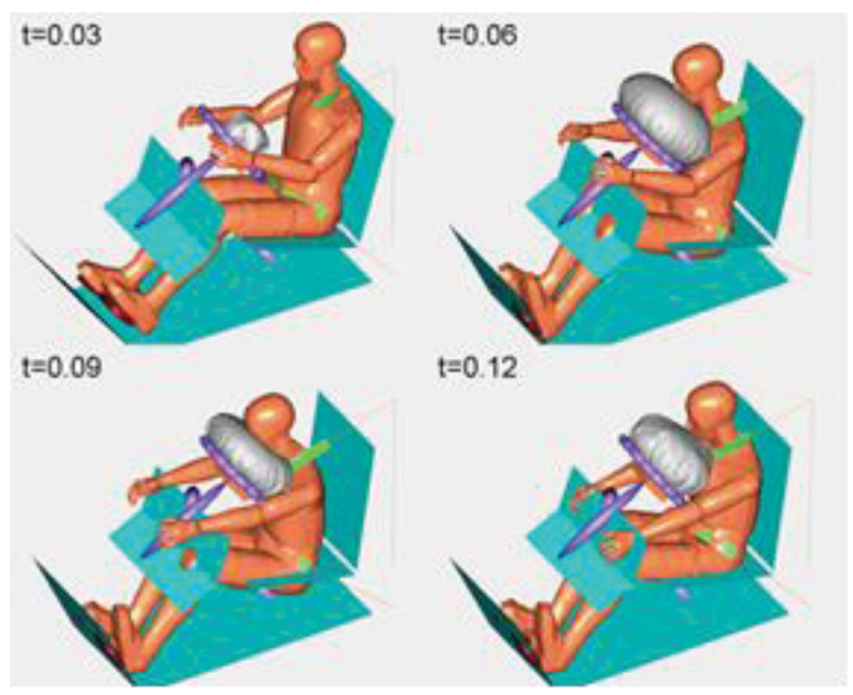

Figure 1: Frontal crash analysis model.

Table 1: Design variables.

\begin{tabular}{llll}
\hline Variable & & Lower limit & Upper limit \\
\hline Airbag & Time to fire [s]/AB_TTF & 0.015 & 0.035 \\
& Mass flow rate/AB_MFR & 0.5 & 2.0 \\
& Vent hole factor/AB_VHF & 0.5 & 2.0 \\
\multirow{3}{*}{ Seatbelt } & Time to fire [s]/SB_TTF & 0.01 & 0.03 \\
& Load limit [N]/SB_LL & 2000 & 6000 \\
Knee bolster & Stiffness factor/KB_SF & 0.5 & 2.0 \\
\hline
\end{tabular}

objective functions. The femoral compressive load, which is significantly lower than the reference criteria in the actual vehicle tests and the analytical models, is treated as a constraint.

\section{ADVANCEMENT OF VEHICLE OCCUPANT RESTRAINT SYSTEM DESIGN}

In this study, in order to improve the design method of vehicle occupant restraint system, it is necessary to reduce the computational load of simulation, to improve the global search capability, to investigate the analysis method to understand the complex interaction between design variables and objective functions, and to integrate these artificial intelligence technologies.

The following three artificial intelligence technologies are integrated and applied to the design of vehicle occupant restraint systems.

(1) Construction of a highly accurate approximate model by machine learning;

(2) Improvement of global search capability by evolutionary multi-objective optimization;

(3) Visualization and knowledge acquisition of multidimensional information using multivariate analysis methods. 
The design exploration flow is shown in Fig. 2. First, a minimum number of calculation samples are obtained by the design of experiments using a crash analysis model, and then a highly accurate approximate model is constructed by machine learning using the samples. Next, the constructed approximate model is used for global search in the design space by evolutionary multi-objective optimization to obtain a Pareto solution set that takes into account the trade-off relationships among the objective functions. Finally, multivariate analysis is performed on the Pareto solution set to visualize the multidimensional information and to obtain useful knowledge for design.

\subsection{Construction of a highly accurate approximate model by machine learning}

In order to perform a global search among a huge number of design variable combinations in the design space, it is required to reduce the computational load. Therefore, we obtained the minimum necessary number of actual calculation samples by design of experiments using the constructed crash analysis model. Then, we constructed a highly accurate approximate model by machine learning using the samples [4].

In this paper, we show the results of constructing approximate models using the Gaussian process [5], which is a kind of machine learning. The approximate model was constructed using the six design variables shown in Table 1 as input variables, the head injury criterion and the chest resultant acceleration as objective functions, and the femoral compressive load as a constraint as output variables, and 100, 200, 300 and 400 actual calculation samples as training data sets.

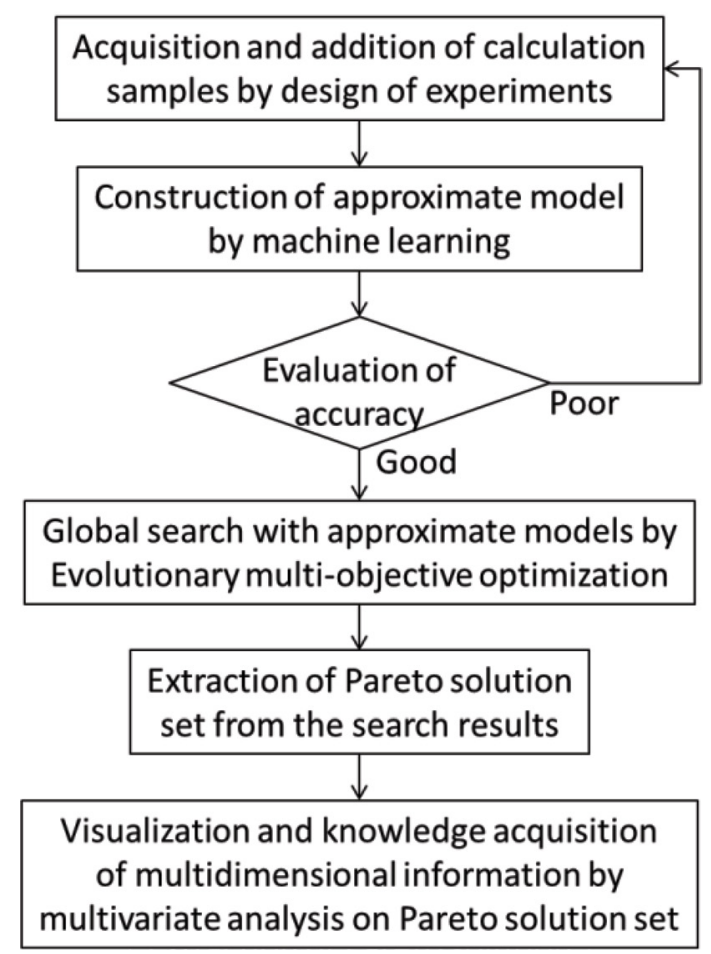

Figure 2: Flow diagram of design exploration. 


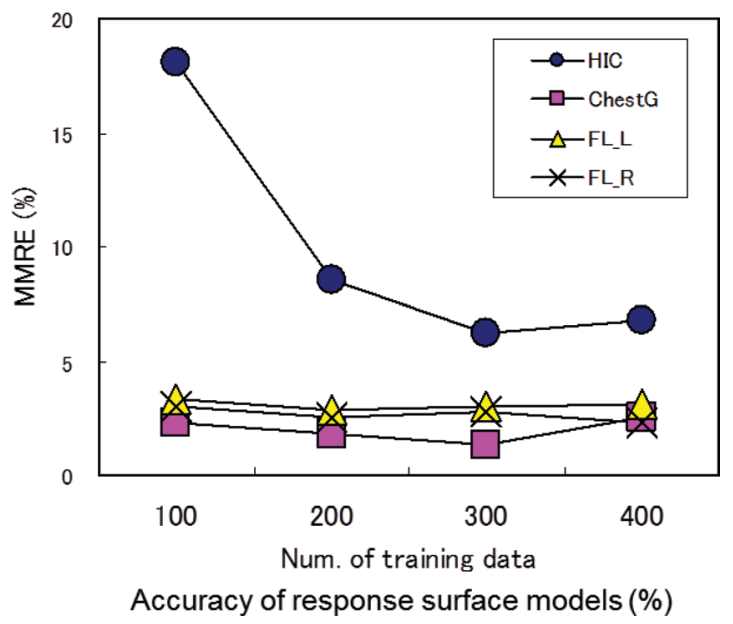

Figure 3: Accuracy evaluation of approximate models.

The accuracy of the approximate models were evaluated using a test data set of 100 points, and it was decided to use the approximate model constructed with 300 samples, which has the best accuracy, in the following design flow. The results of the accuracy evaluation of the approximate models are shown in Fig. 3.

\subsection{Improvement of global search capability by evolutionary multi-objective optimization}

In this study, the adaptive range multi-objective genetic algorithm (ARMOGA) [6], which is one of the evolutionary multi-objective optimization methods with excellent global search capability, was used to optimize the multi-objective design of a vehicle occupant restraint system [7]. The flow diagram of the ARMOGA is shown in Fig. 4. The ARMOGA adaptively changes the solution search region for a certain number of generations. In the normal generation, the general genetic operator is applied, and in the domain-adaptive generation, the mean and variance of each design variable in the archive population obtained so far are calculated, and the search population is reinitialized in the domain adapted to the distribution to achieve efficient search. In addition, fitness sharing is used to maintain diversity, and the search capability is high for multi-modal problems.

The problem was defined as a two-objective optimization problem with six design variables as shown in Table 1, where the head injury criterion and the chest resultant acceleration were the objective functions and the femoral compressive load was the constraint. Ten trials of optimization were conducted by ARMOGA with different initial populations. The parameter settings of ARMOGA are shown in Table 2. The number of search generations was set to 50 generations, where the convergence of the Pareto surface progression was confirmed. The population size $40 \times 50$ generations $\times 10$ trials, which took 20,000 evaluations in total, and the approximate model is substituted to achieve fast solution search.

Figure 5 shows the results of plotting all 20,000 points explored in the optimization calculation on the objective function space. The green points are feasible solutions that satisfy the safety criteria, and the orange points are infeasible solutions that do not satisfy the safety criteria. The Pareto solution sets of the two objective functions are extracted from the search 


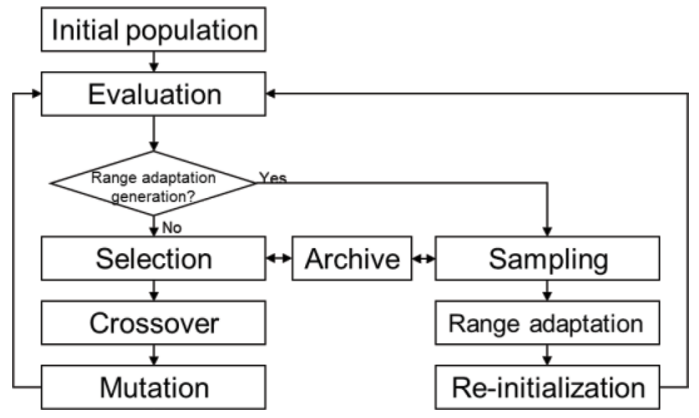

Figure 4: Flow diagram of ARMOGA.

Table 2: Parameter setting of ARMOGA.

\begin{tabular}{ll}
\hline Genetic parameters & \\
\hline Population size & 40 \\
Num. of generation & 50 \\
Crossover & BLX-0.5 \\
Crossover rate & 1.0 \\
Mutation rate & 0.1 \\
\hline Range adaptation operation & \\
\hline Starting generation & 20 \\
AR interval & 5 \\
\hline
\end{tabular}

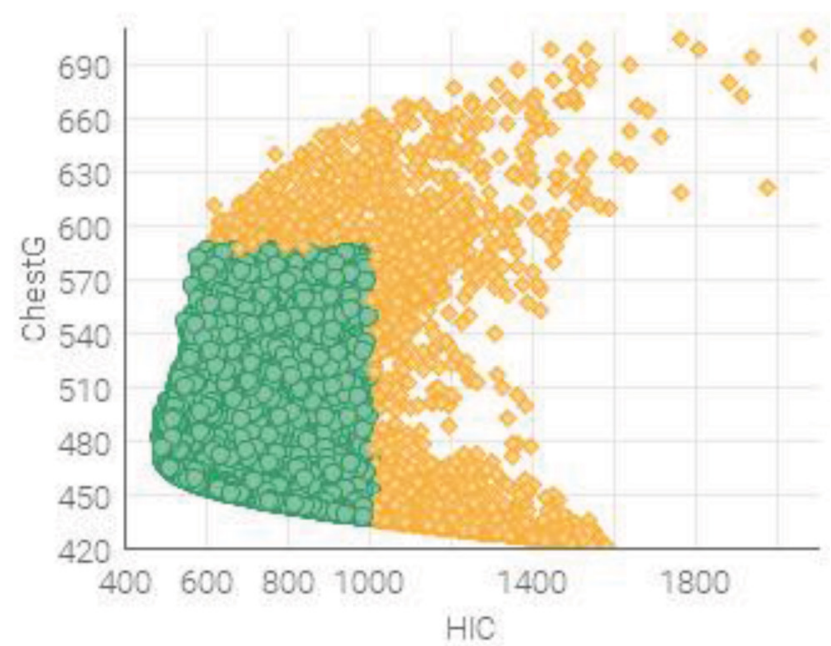

Figure 5: All solutions obtained by ARMOGA (green: feasible solutions, orange: infeasible solutions). 


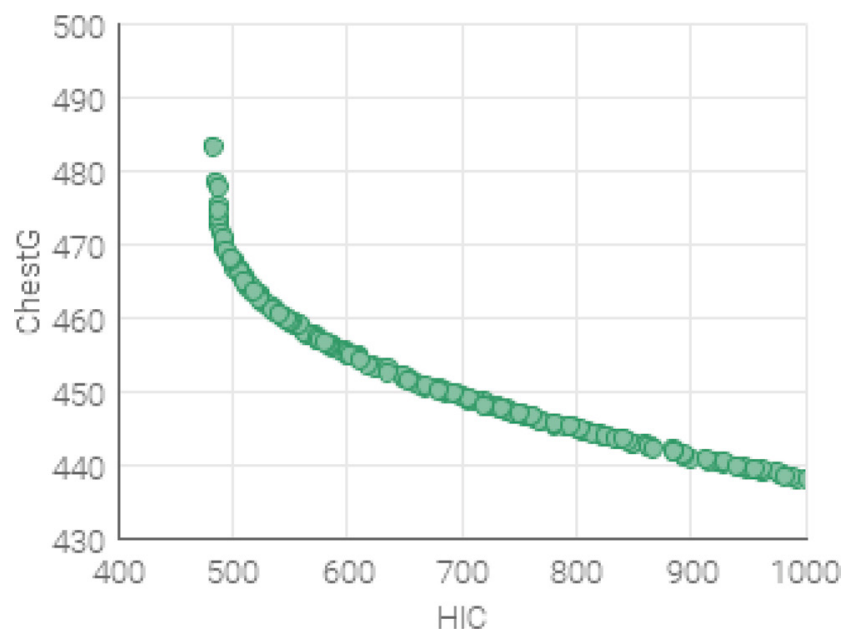

Figure 6: Pareto solutions obtained by ARMOGA.

results and plotted in Fig. 6. Multivariate analysis was applied to a total of 214 extracted Pareto solutions to visualize the multidimensional information and to obtain useful knowledge for design.

3.3 Visualization and knowledge acquisition of multidimensional information using multivariate analysis methods

Multivariate analysis was applied to 214 Pareto solutions extracted by global search with evolutionary multi-objective optimization to visualize multidimensional information and to acquire knowledge useful for design. First, cluster analysis was applied to the Pareto solution set, and each cluster was visualized by self-organizing maps, and the interaction between the design variables and the objective functions was revealed by analysing the characteristics of each cluster.

\subsubsection{Cluster analysis}

Cluster analysis [8] classifies each solution in the data set to be analysed into a number of clusters based on the similarity between them. By analysing the characteristics of the classified clusters and the differences between clusters, useful knowledge for design can be obtained.

In this study, the Ward method, which is one of the hierarchical cluster analysis methods, was used to analyse the clusters of the Pareto solution set. The tree diagram obtained by the cluster analysis is shown in Fig. 7. Summary of the clusters is shown in Table 3; 214 Pareto solutions were classified into four clusters. Cluster 0 contains 51 solutions, cluster 1 contains 67 solutions, cluster 2 contains 56 solutions and cluster 3 contains 40 solutions. The Pareto solutions are plotted in the objective function space, colour-coded by cluster, and the results are shown in Fig. 8. The similarity between the clusters shown in the tree diagram in Fig. 7, and it can be confirmed that the clusters are firstly divided into two large clusters, clusters 0 and 1 , and clusters 2 and 3, and that each large cluster is further divided into two small clusters. In addition, it can be confirmed that the clusters are aligned in the objective function 


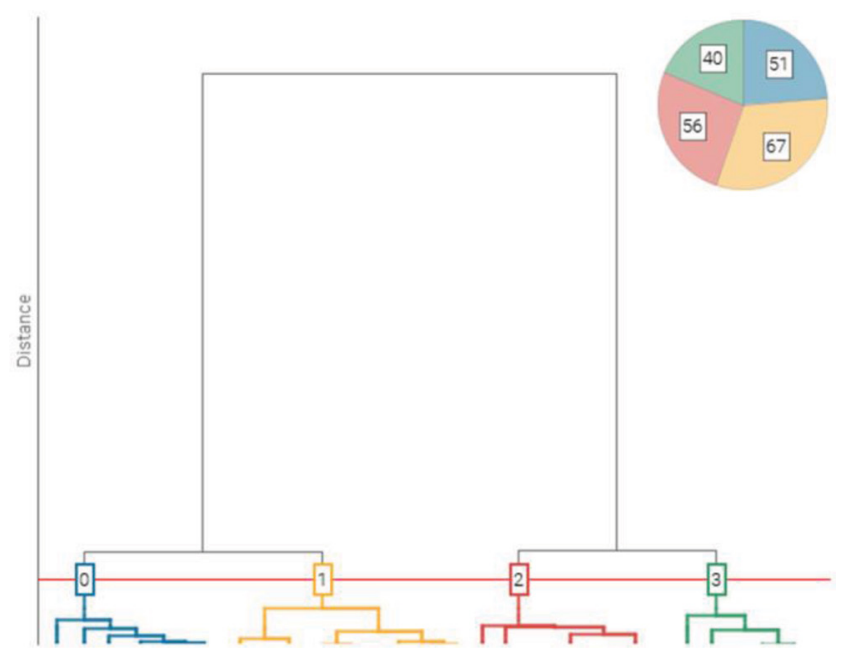

Figure 7: Tree diagram by cluster analysis (Ward method).

Table 3: Summary of clusters.

\begin{tabular}{|c|c|c|c|c|}
\hline & Cluster 0 & Cluster 1 & Cluster 2 & Cluster 3 \\
\hline \multirow{3}{*}{ Num. of solutions } & \multicolumn{4}{|c|}{214} \\
\hline & \multicolumn{2}{|c|}{118} & \multicolumn{2}{|c|}{96} \\
\hline & 51 & 67 & 56 & 40 \\
\hline
\end{tabular}

space in Fig. 8. Therefore, it is considered that the factors controlling the trade-off relationship of the Pareto solution can be clarified by analysing the characteristics of each cluster.

\subsubsection{Self-organizing maps}

Self-organizing maps (SOM) is a kind of competitive learning neural network, which maps the similar vectors to the neighbourhood and the dissimilar vectors to the distance on the two-dimensional map by learning the multidimensional vector population [9]. Based on the mapped results, it is possible to analyse and understand the common attributes of the populations gathered in the neighbourhood and the differences between distant populations, and to analyse the correlation of multidimensional information efficiently.

In this study, based on the results of the cluster analysis of the Pareto solution set by Ward's method, the factors constituting each cluster were analysed by SOM. As shown in Fig. 7, in the cluster analysis, the Pareto solution set is divided into two large clusters, and each large cluster is further divided into two small clusters. Therefore, we created SOM maps for the whole Pareto solution set, for clusters 0 and 1, for clusters 2 and 3, and for clusters $0,1,2$ and 3 , respectively.

Figure 9 shows the overall diagram of the SOM maps we have created. The solutions composing the Pareto solution set have the information of 8 variables ( 8 dimensions) of 6 design variables +2 objective functions, and similar solutions are placed near each other on the 2-dimensional map, and dissimilar solutions are placed far from each other. Each SOM 


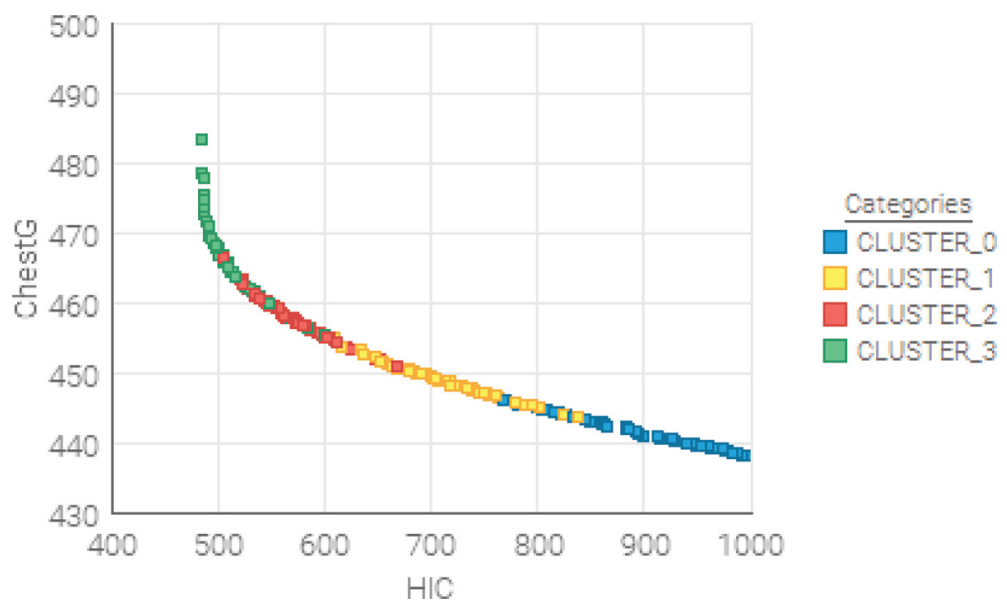

Figure 8: Pareto solutions with colour-coded by cluster plotted in objective function space.

map in Fig. 9 displays a colour contour plot of the 8 variable values of the design variables or objective functions of the placed solutions, and also places the colour contour plots on a two-dimensional map based on the similarity between the 8 variables. Each SOM map allows us to visually grasp the correlation among variables in each Pareto solution set. The following correlations can be identified from these SOM maps.

- The map (a) of all Pareto solutions shows that for the two objective functions, HIC and Chest $G$, and the design variable $A B \_M F R$, the correlation between $H I C$ and $A B \_M F R$ is negative, and between $C h e s t G$ and $A B \_M F R$ is positive. For the other design variables, no correlations with other variables can be identified.

- The map (b) of clusters 0 and 1 shows the correlation among the three design variables, $A B \_T T F, A B \_V H F$ and $S B \_T T F$, and the correlation between the two design variables, $S B \_L L$ and $K B \_S F$, in addition to the correlation among the two objective functions, HIC and $C h e s t G$, and the design variable $A B \_M F R$.

- The map (c) of cluster 0 shows the correlation between the two design variables, $A B \_T T F$ and $S B \_L L$, in addition to the correlation among the two objective functions, $H I C$ and Chest $G$, and the design variable $A B \_M F R$.

- The map (d) of cluster 1 shows the correlation between the two design variables, $A B \_T T F$ and $A B_{-} V H F$, in addition to the correlation among the two objective functions, $H I C$ and Chest $G$, and $A B \_M F R$.

- The map (e) of clusters 2 and 3 shows the correlation among the three design variables, $A B \_V H F, S B \_T T F$ and $S B \_L L$, in addition to the correlation among the two objective functions, $H I C$ and $C h e s t G$, and the design variable $A B \_M F R$.

- The map (f) of cluster 2 shows the correlation among the three design variables, $A B \_V H F$, $S B \_T T F$ and $S B \_L L$, and the correlation between the two design variables, $A B \_T T F$ and $K B \_S F$, in addition to the correlation among the two objective functions, $H I C$ and $C h e s t G$, and the design variable $A B \_M F R$.

- The map (g) of cluster 3 shows the correlation among the four design variables, $A B \_T T F$, $A B \_V H F, S B \_T T F$ and $K B \_S F$, in addition to the correlation among the two objective functions, $H I C$ and $C h e s t G$, and the design variable $A B \_M F R$. 


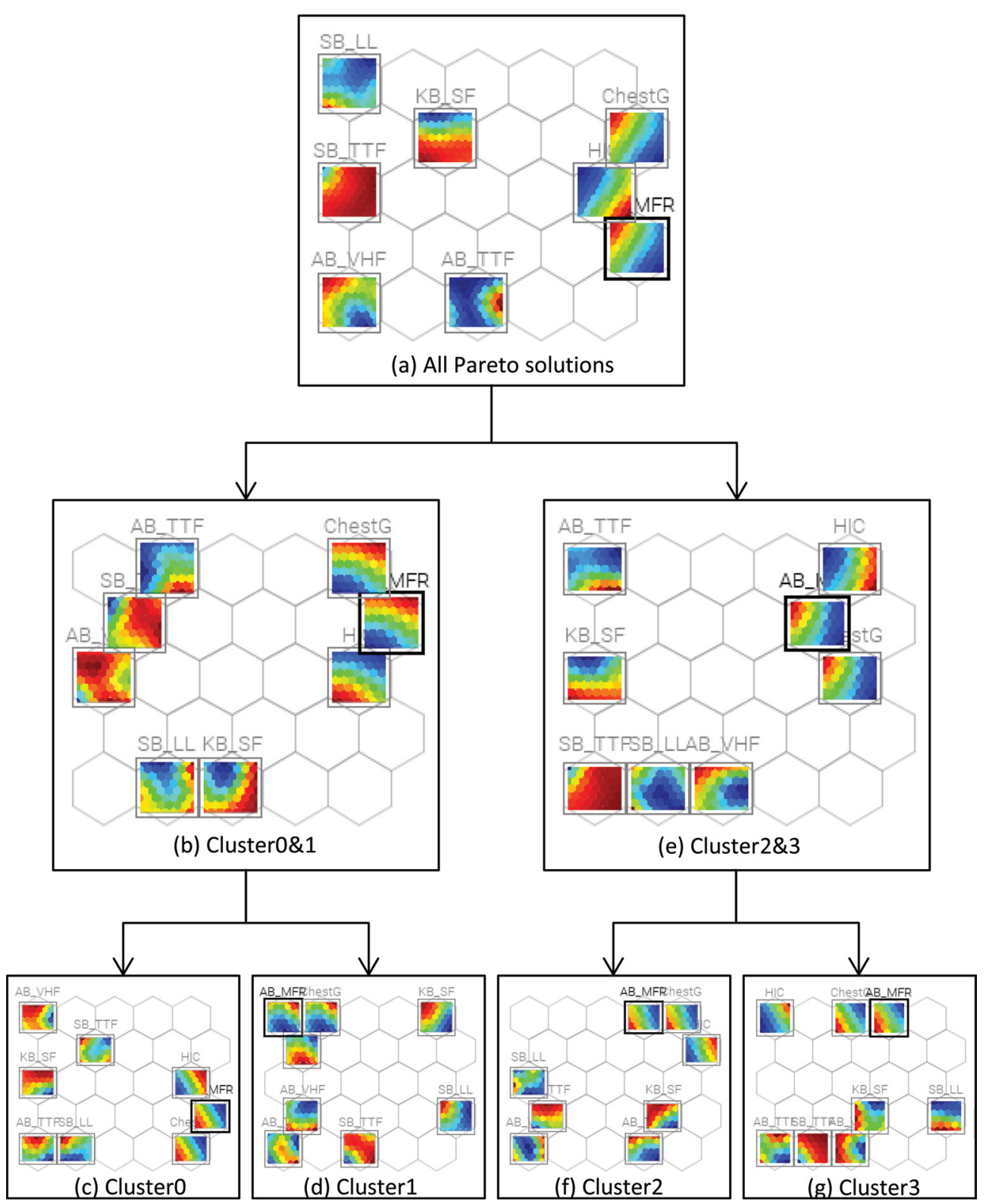

Figure 9: Overall diagram of SOM maps of each Pareto solution set.

From these results and the cluster analysis results plotted on the objective function space shown in Fig. 8, the factors controlling the trade-off of the two objective functions HIC and Chest $G$ in each cluster can be visually understood, and quantitative design information can be obtained by making hypotheses based on the visual information and verifying them by statistical analysis. When the colour distribution of the colour contour diagram of the variables whose correlation has been confirmed is similar, such as the correlation between the two objective functions $H I C$ and $C h e s t G$ and the design variable $A B \_M F R$ confirmed in each Pareto solution 
Table 4: Summary of correlation among design variables and objective functions ( $\odot$ : positive linear, $\bullet$ : negative linear, 凉: non-linear).

\begin{tabular}{|c|c|c|c|c|c|c|c|c|}
\hline & \multicolumn{3}{|l|}{ Airbag } & \multicolumn{2}{|l|}{ Seatbelt } & $\begin{array}{l}\text { Knee } \\
\text { bolster }\end{array}$ & \multicolumn{2}{|c|}{$\begin{array}{l}\text { Objective } \\
\text { function }\end{array}$} \\
\hline & AB_TTF & AB_MFR & AB_VHF & SB_TTF & SB_LL & KB_SF & $\mathrm{HIC}$ & ChestG \\
\hline \multirow[t]{2}{*}{$\begin{array}{l}\text { (a) All Pareto } \\
\text { solutions }\end{array}$} & & O & & & & & $\bullet$ & O \\
\hline & 榢 & & 象 & 夈 & & & & \\
\hline \multirow[t]{2}{*}{ (b) Cluster0\&1 } & & & & & O & O & & \\
\hline & & 0 & & & & & - & 0 \\
\hline \multirow{2}{*}{ (c) Cluster 0} & O & & & & O & & & \\
\hline & & 0 & & & & & - & 0 \\
\hline \multirow{2}{*}{ (d) Cluster 1} & 安 & & 串 & & & & & \\
\hline & & O & & & & & - & 0 \\
\hline \multirow{3}{*}{ (e) Cluster $2 \& 3$} & & & O & $\bullet$ & O & & & \\
\hline & & O & & & & & - & 0 \\
\hline & & & 0 & - & 0 & & & \\
\hline \multirow[t]{2}{*}{ (f) Cluster 2} & 0 & & & & & $\circ$ & & \\
\hline & & O & & & & & $\bullet$ & O \\
\hline \multirow{2}{*}{ (g) Cluster 3} & 棕 & & - & - & & 淁 & & \\
\hline & & 0 & & & & & - & 0 \\
\hline
\end{tabular}

set, it means that there is a linear correlation between the variables. On the other hand, if the correlations are confirmed but the distribution of colours in the colour contour plot is not similar, it means that there is a non-linear correlation between those variables. In this case, it can be understood that it is necessary to verify the interaction between the variables in order to control the trade-off between the two objective functions HIC and Chest $G$ in that region. The correlations among design variables and objective functions are summarized in Table 4.

Finally, we examine the significance and insignificance of the design variables. Since all the design variables are correlated in some cluster, we can conclude that they are significant. On the other hand, if they are fixed at almost constant values in a particular cluster, i.e. high or low values overall, such as $A B \_V H F, S B \_T T F$ and $S B \_L L$ in the map (e), then we can conclude that they are less significant in controlling the trade-off between the objective functions in that cluster.

\section{CONCLUSION}

In this study, in order to improve the design method of vehicle occupant restraint systems, the following three artificial intelligence technologies were integrated and applied to the design of a vehicle occupant restraint system. 
(1) Construction of a highly accurate approximate model by machine learning;

(2) Improvement of global search capability by evolutionary multi-objective optimization;

(3) Visualization and knowledge acquisition of multidimensional information using multivariate analysis methods.

First, we constructed a highly accurate approximate model by machine learning. Then, using the approximate model, the global search was performed by evolutionary multi-objective optimization to obtain the Pareto solution set considering the trade-off relationship between the objective functions. Finally, multivariate analysis using cluster analysis and self-organizing maps was performed on the Pareto solution set. These multivariate analysis provided visual information on the factors controlling the trade-offs among the objective functions and the interactions among the design variables.

\section{ACKNOWLEDGEMENT}

This work was partly supported by a grant from the Suzuki Foundation.

\section{REFERENCES}

[1] Obayashi, S., Jeong, S. \& Chiba, K., Multi-objective design exploration for aerodynamic configurations, AIAA-2005-4666, 2005.

[2] Natori, S. \& Yu, Q., An Application of CAP (Computer-Aided Principle) to Structural Design for Vehicle Crash Safety, SAE Technical Paper 2007-01-0882, 2007.

[3] Deb, K., \& Srinivasan, A., Innovization: innovative design principles through optimization, Proceedings of the 8th annual conference on Genetic and evolutionary computation, pp.1629-1636, 2006.

[4] Horii, H., Estimate modelling for assessing the safety performance of occupant restraint systems, WIT Transactions on the Built Environment, Vol.134, pp.627-635, 2014.

[5] Rasmussen, C.E. \& Williams, C.K.I., Gaussian Processes for Machine Learning, MIT Press, 2006.

[6] Sasaki, D. \& Obayashi, S., Efficient search for trade-offs by adaptive range multiobjective genetic algorithms, AIAA Journal of Aerospace Computing, Information and Communication, 2, pp. 44-64, 2005.

[7] Horii, H., Multi-objective optimization of vehicle occupant restraint system by using evolutionary algorithm with response surface model, International Journal of Computational Methods and Experimental Measurements, 5(2), pp. 163-173, 2017.

[8] Murtagh, F. \& Legendre, P., Ward's hierarchical clustering method: clustering criterion and agglomerative algorithm, Journal of Classification, 31(3), pp. 274-295, 2014.

[9] Kohonen, T., Self-Organizing Maps, Springer-Verlag, 2001. 